\title{
The Introduction of Specific Water and Maximum Airborne Specific Water \& the Improvement of Dynamic Equations on Non-Uniform Saturated Moist Atmosphere
}

\author{
Xingrong Wang*, Yan Feng \\ Anhui Key Lab of Atmospheric Science and Satellite Remote Sensing, Anhui Meteorology Institute, Hefei, China

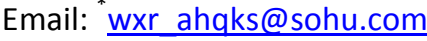

Received 2 April 2015; accepted 28 June 2015; published 1 July 2015

Copyright (C) 2015 by authors and Scientific Research Publishing Inc. This work is licensed under the Creative Commons Attribution International License (CC BY). http://creativecommons.org/licenses/by/4.0/

(c) (i) Open Access

\section{Abstract}

To eliminate the irrational supposition that condensed liquid water always falls immediately, specific water $m$ and maximum airborne specific water $m_{m}$ are introduced into the dynamic framework on non-uniform saturated moist atmosphere $(m$ is the ratio of the airborne liquid water mass to the moist air mass in unit cubage moist air, $m_{m}$ is its maximum value with $m_{m}=\gamma w^{1.5} q_{s}$, $\gamma, w$ and $q_{s}$ are airborne coefficient, vertical velocity and saturated specific humidity respectively). The balance equation between water vapor and airborne liquid water is derived. From the balance equation, a new formula of precipitate rate is got. The analysis shows that in the air stream with some upward vertical velocity $(w \geq 0)$, the condensed liquid water can precipitate under the condition with $q=q_{s}$ ( $q$ is specific humidity) and $m=m_{m}=\gamma w^{1.5} q_{s}$ only, otherwise it is detained in the air and becomes airborne liquid water. Not only does precipitating liquid water contain condensed liquid water, but also contains converged and existing airborne liquid water. Following above discussion, improved dynamic equations on non-uniform saturated moist atmosphere are provided.

\section{Keywords}

Airborne Liquid Water, Specific Water, Maximum Airborne Specific Water, Airborne Coefficient, Dynamic on Non-Uniform Saturated Moist Atmosphere

\footnotetext{
${ }^{*}$ Corresponding author.

How to cite this paper: Wang, X.R. and Feng, Y. (2015) The Introduction of Specific Water and Maximum Airborne Specific Water \& the Improvement of Dynamic Equations on Non-Uniform Saturated Moist Atmosphere. Atmospheric and Climate Sciences, 5, 245-256. http://dx.doi.org/10.4236/acs.2015.53018
} 


\section{Introduction}

Due to the important roles of water vapor in the genesis and development of weather systems, many studies have contributed to the dynamical discussion on moist atmosphere, e.g., Betts A. K., (1973) [1]; Xie Y. B., (1978) [2]; Wang L. M., (1980) [3] [4]; Tripoli G. J., W. R. Cotton, (1981) [5]; Pointin Y., (1984) [6]; Hauf T., H. Holler, (1987) [7]; Ooyama K. V., (1990) [8]; (2000) [9]; Persson O., (1995) [10]; Wu G. X., Y. P. Cai and X. J. Tang, (1995) [11]; Soriano L. J., E. L. Diez, (1997) [12]; Schubert W. H., S. A. Hausman, and M. Garcia et al., (2001) [13]; Bannon P. R., (2002) [14].

To consider the transitional area between the unsaturated and saturated atmosphere, the discontinuity could occur in the latent heat term of the thermodynamic equation when the latent heat is released in the saturated atmosphere whereas latent heat is not released in the unsaturated atmosphere. Traditionally, the discontinuity in the latent heat term of the thermodynamic equation can be summarized by the Dirac delta function. The discontinuity makes the theoretical analysis very difficult for near saturated atmosphere, where the relative humidity is usually large enough to condensate but not saturated (just like rain regions), so these discussions are limit to the condition of saturated atmosphere e.g., Wang L. M., (1980) [3] [4]; Wu G. X., Y. P. Cai and X. J. Tang, (1995) [11], (realistic atmosphere is non-uniform saturated), or limit to the condition of equilibrium airflow, e.g., Ooyama K.V., (1990) [8]; (2000) [9]; Schubert W.H., S.A. Hausman, and M. Garcia et al., (2001) [13], (realistic airflow is not always equilibrium).

However, the condensation process does not occur so abruptly in realistic atmosphere. According to the observational evidences pointed by Mason, (1971) [15], the condensation process might occur at 78\% of relative humidity under the condition of the appropriate condensation nuclear in real atmosphere. Therefore, a condensation probability function $\left(q / q_{s}\right)^{k}$ is introduced by Wang X. R. and K. J. Wu, (1995) [16], Wang X. R., C. E. Chi and Z. X. Wang, (1997) [17] and Wang X. R., Z. X. Wang, and C. N. Shi, (1999) [18], which is expected to represent the continuity of this kind of variation, here $q$ and $q_{s}$ are the specific humidity and saturated specific humidity respectively, $k$ is a function of pressure, temperature, specific humidity and the density of condensation cores. When $k \rightarrow \infty$, the function is degraded to the Heaviside step function. Thus, the condensation probability function is a generalized Heaviside step function, which leads to the continuity of the latent heat term of the thermodynamic equation in the transition area between the unsaturated and saturated air. They constituted a more approximate thermodynamic equation for the saturated and/or near saturated atmosphere, where the relative humidity is usually large enough to condensate but not saturated (just like rain regions). Based on the fact that condensation occurs when $\left(q / q_{s}\right)>0.78$ in the real atmosphere due to the existence of condensation cores, and by theoretical analysis and numerical simulation, Wang X. R., Z. X. Wang, and C. N. Shi, (1999) [18], found when $k$ is about 9 , the condensation in the atmosphere is represented well. And hence, we specify $k=9$ in our study. Following this discussion, the dynamic equations on non-uniform saturated moist atmosphere (EQSNUSMA) are provided.

On the basis of EQSNUSMA, a battery of results is got:

Wang X. R. and K. J. Wu, (1995) [16], found that the direction of geo-strophic equilibrium wind of moist air is parallel to equipotential line, its wind speed is equal to the product of dry air geo-strophic equilibrium wind speed and static non-equilibrium parameter $\left[\exp \left(-\left(L q_{s} / C_{p} T\right)\left(q / q_{s}\right)^{k}\right)\right]$. The wind speed of geo-strophic equilibrium wind of moist air is less than that of dry air, because static non-equilibrium parameter is less than 1. It was pointed out that the super-geo-strophic behavior of low level jet streams is caused by non-equilibrium relating to jet strengthening, it is not equilibrium airflow.

The non-uniform saturated moist potential vorticity (NUSMPV), or the generalized moist potential vorticity (GMPV), is thus defined and its tendency equation is derived by Wang X. R., Z. X. Wang and C. N. Shi, (1998) [19]; Gao S. T., X. R. Wang and Y. S. Zhou, (2004) [20]; Wang X. R. and M Wei, (2007) [21]. The analysis shows that in a frictionless and moist adiabatic flow, the NUSMPV, (or GMPV), can be generated in the unsaturated air only and its generation rate is determined by the environmental moisture and its gradient. It is further shown that in the sub-saturated regions $\left(1>\left(q / q_{s}\right)>0.78\right)$, the NUSMPV is not conserved; whereas, in the other unsaturated regions, the NUSMPV may be nearly conserved.

Based on the works by Wang X. R., Z. X. Wang and C. N. Shi, (1998) [19]; Wang X. R., X. P. Chen and S. P. Xun, (1998) [22]; Wang X. R., K. J. Wu and X. P. Chen et al., (1999) [23] and Wang X. R., Z. X. Wang, and C. N. Shi, (1999) [18], the transformation mechanism between weather systems of different scale is discussed by Wang X. R., M. Wei, (2007) [21]. According to the relativity principle of conservation, by discussing the 
non-dimensional form of the NUSMPV equation, the atmospheric motion is classified into three types: motions with conserved, quasi-conserved, and non-conserved NUSMPV. It is pointed out as follows. When the dynamic non-equilibrium degree $(A+B) / P_{0}$ decreases so that the condition of NUSMPV conservation is satisfied, the atmospheric motion in NUSMPV conservation state transforms from smaller scale to larger scale mainly by the very fast adaptation process (A is solenoidal term, B is diabetic heating, $P_{0}$ is narrow sense NUSMPV.). In this kind of process the non-equilibrium energy is dispersed and lost by gravitational and sound wave. On the contrary, when the $(A+B) / P_{0}$ increases so that the condition of non-conservation is satisfied, the atmospheric motion in the NUSMPV non-conservation state transforms from larger scale to smaller scale mainly by the very fast excitation process. In this kind of process the $(\mathrm{A}+\mathrm{B})$ changes NUSMPV by changing vorticity and $\nabla \theta^{*}$. Either of the two kinds of transformation process mentioned above will constantly go along till the atmospheric motion is back in the state of NUSMPV quasi-conservation. From the transformation mechanism between weather systems of different scale, according to the basal characteristic of the sudden heavy rain in mid-latitude (SHR), by discussing the condition of NUSMPV non-conservation, the four necessary occurrence conditions of SHR are proved theoretically by Wang X. R., Y. Y. Zheng and S. T. Gao et al., (2006) [24]. By the analysis of the Doppler radar and cloud chart data related to two courses of the SHR in Hefei city in 1999, it is known that, just before occurrence of SHR, both courses have two common possible premonitory characters embodying the four necessary conditions.

Furthermore, in recent years, some new variables from EQSNUSMA, such as generalized potential temperature, GMPV, generalized convective vorticity vector et al., are applied more to the dynamical analysis of hot and humid weather systems and torrential rain systems, e.g., Gao S. T., Y. S. Zhou, T. Lei et al. (2005) [25]; Sheng S. Q. and Y. S. Zhou, (2007) [26]; Yang S., S. T. Gao, D. H. Wang, (2007) [27]; Zhou Y. S., J. Cao and D. H. Wang. (2007) [28]; Duan T. Y., G. Deng. and D. H. Wang, (2007) [29]; Gao S. T. and C. G. Cui, (2007) [30]; Cao J. and S. T. Gao, (2008) [31]; Zhou Y. S., (2009) [32]; Zhaoming Liang, Chungu Lu and Edward I. Tollerud, (2010) [33]; Zhou Y. S. and K. F. Zhu. (2010) [34].

Such great progress for dynamic on non-uniform saturated moist atmosphere has been made in theory and application aspects, but, in all studies, almost no one is involved in the discussion on condensed liquid water. Traditionally, it is supposed that condensed liquid water always precipitates immediately. However, it is not true, in realistic atmosphere, the condensed liquid water either falls down in the form of precipitate or is detained in the air in the form of airborne liquid water (fog or cloud). By the analysis of some failure model experiments on dynamic equations on non-uniform saturated moist atmosphere, it is found that the reason of failure is exactly the irrational supposition that condensed liquid water always falls down immediately. So, in the dynamic equations on non-uniform saturated moist atmosphere, it becomes a question how to consider the condensed liquid water in air. By the discussion of airborne liquid water content and the balance equation between water vapor and liquid water, the new idea (not only precipitate contains condensed liquid water also includes airborne liquid water) is firstly raised and an advanced precipitable formula is set up by Wang, X.R., Gao, S.T. (2007) [35]. But the paper is not involved in the discussion on the condition, under which condensed liquid water can precipitate only, otherwise it is detained in the air and becomes airborne liquid water. And that, in the paper, the deduction in $p$ coordinate is not correct, so the airborne coefficient estimated is also an inaccuracy.

To eliminate the irrational supposition that condensed liquid water always falls down immediately, specific water $m$ and maximum airborne specific water $m_{m}$ is introduced into the dynamic framework to derive the balance equation between water vapor and airborne liquid water and a new formula of precipitate rate in Section 2. Following above discussion, improved dynamic equations on non-uniform saturated moist atmosphere are provided in Section 3. From the data provided by the leading group for studying the super-heavy rain in Henan in China in August 4-8 1975 [LGSHR1975] (1977), airborne coefficient is roughly estimated in Section 4. The summary is given in Section 5.

\section{The Balance Equation between Water Vapor and Airborne Liquid Water}

\subsection{The Discussion in the Condition of $w \geq 0$}

In the condition of $w \geq 0$ ( $w$ is the vertical velocity in $\mathrm{z}$ coordinate), if the water vapor condenses, the condensed liquid water falls down in the form of precipitate or is detained in the air in the form of airborne liquid water (fog or cloud). Because of the resistance of up-current, if condensed liquid water does not gain enough mass, (larger water droplets overcoming up-current resistance are not able to occur,) it is detained in the air in 
the form of airborne liquid water. If it gains enough mass, (larger water droplets overcoming up-current resistance are able to occur,) the liquid water mass over the maximum airborne liquid water mass detained by up-current falls down in the form of precipitate.

Following Wang X. R., Z. X. Wang and C. N. Shi (1999) [18], by introducing the condensation probability function $\left(q / q_{s}\right)^{k}$ to replace the Dirac delta function, the thermodynamic equation and the balance equation between water vapor and airborne liquid water may be respectively expressed as

$$
\begin{gathered}
C_{p} \frac{\mathrm{d} T}{\mathrm{~d} t}-\frac{1}{\rho} \frac{\mathrm{d} p}{\mathrm{~d} t}=-L_{d} \frac{\mathrm{d}}{\mathrm{d} t}\left[\left(\frac{q}{q_{s}}\right)^{k} q_{s}\right]+Q_{d} \\
\begin{cases}-\frac{\mathrm{d}}{\mathrm{d} t}\left[\left(\frac{q}{q_{s}}\right)^{k} q_{s}\right]=\frac{\mathrm{d} m}{\mathrm{~d} t} & m<m_{m} \\
-\frac{\mathrm{d}}{\mathrm{d} t}\left[\left(\frac{q}{q_{s}}\right)^{k} q_{s}\right]=\frac{\mathrm{d} m}{\mathrm{~d} t}+\eta & m=m_{m}\end{cases}
\end{gathered}
$$

where $k=9, Q_{d}$ is the diabatic heating excluding the latent heating, $m$ is specific water (the ratio of the airborne liquid water mass to the moist air mass in unit cubage moist air), $m_{m}$ is maximum airborne specific water (the ratio of the maximum airborne liquid water mass detained by up-current to the moist air mass in unit cubage moist air), $\eta$ is precipitate rate (the ratio of the liquid water mass over the maximum airborne liquid water mass to the moist air mass in unit cubage moist air), others are common symbols.

Following Mason, (1971) [15], $m_{m}$ may be expressed as

$$
m_{m}=\frac{4}{3} \pi \frac{\rho_{w}}{\rho} \int \frac{\mathrm{d} n(r)}{\mathrm{d} r} r^{3} \mathrm{~d} r
$$

where $\rho_{w}$ is water density, $\rho$ is air density, $n(r)$ is the number of liquid water droplets with radius $r$ in unit cubage moist air. Following Levin, 1954 [36], $n(r)$ may be expressed as

$$
n(r)=\frac{N}{\sqrt{2 \pi} \log \sigma_{g}} \exp \left[-\frac{\left(\log r-\log r_{g}\right)^{2}}{2 \log ^{2} \sigma_{g}}\right]
$$

where $N$ is the all number of liquid water droplets, $\sigma_{g}$ is standard deviating, $r_{g}$ is geometrical mean radius (the radius of liquid water droplets with the highest probability. From Equation (4), we have

$$
\int \frac{\mathrm{d} n(r)}{\mathrm{d} r} r^{3} \mathrm{~d} r=r_{g}^{3} N\left[3 \ln ^{2} \sigma_{g}+\frac{2 \sqrt{2}}{\sqrt{\pi}} \ln \sigma_{g}\right] \exp \left(4.5 \ln ^{2} \sigma_{g}\right)
$$

According to the observational evidences pointed by Mason, (1971) [15], Squires P. (1958) [37], Warner J. (1955) [38], Warner J. and T. D. Newnham, (1952) [39] and Weickmann H. K. and H. J. Aufm Kampe, (1953) [40], The distribution of airborne liquid water mass in cloud is related to the distribution of vertical velocity. According to force equilibrium principle, in the condition of $w \geq 0$, if the radius of liquid water droplets is smaller, liquid water droplets uptrend, on the contrary downtrend. So when $m=m_{m}$, the liquid water droplet with $r_{g}$ is in the state of equilibrium, its viscous resistance is equal to its gravity, so

$$
6 \pi \mu r_{g} w_{g}=\frac{4}{3} \pi r_{g}^{3} \rho_{w} g .
$$

where $\mu$ is coefficient of viscosity, $g$ is acceleration of gravity, $w_{g}$ is its velocity relative to up-current. Because it is in the state of equilibrium, so

$$
w_{g}^{2}=w^{2}
$$

With Equation (5), (6) and (7), Equation (3) becomes 


$$
\begin{gathered}
m_{m}=f\left(\sigma_{g}\right) N w^{1.5} \\
f\left(\sigma_{g}\right)=6 \sqrt{4.5} \pi \frac{\mu^{1.5}}{\rho \rho_{w}^{0.5} g^{1.5}}\left[3 \ln ^{2} \sigma_{g}+\frac{2 \sqrt{2}}{\sqrt{\pi}} \ln \sigma_{g}\right] \exp \left(4.5 \ln ^{2} \sigma_{g}\right) .
\end{gathered}
$$

From the physical properties in cumuliform clouds versus height above cloud base (Figure 1) by Weickmann H. K. and H. J. Aufm Kampe, (1953) [40], it may be found that, if the relation curve $T_{b}$ - $h$ of the temperature at observation point $\left(T_{b}\right)$ to the height above base $(h)$ convert to the $q_{s}-h$ relation curve, it is very similar to the relation curve $N$-h of the number of cloud droplets in cumulus clouds $(N)$ to $h$, in other words, $N$ is roughly proportional to $q_{s}$, so in saturated moist air,

$$
N=a q_{s}
$$

here $a$ is the coefficient independent of $p$ and $T$. Because $m_{m}$ may be satisfied in saturated atmosphere only, with Equation (10), Equation (8) and (9) become:

$$
\begin{gathered}
m_{m}=\gamma\left(a, \sigma_{g}\right) w^{1.5} q_{s} \\
\gamma\left(a, \sigma_{g}\right)=6 \sqrt{4.5} \pi \frac{\mu^{1.5} a}{\rho \rho_{w}^{0.5} g^{1.5}}\left[3 \ln ^{2} \sigma_{g}+\frac{2 \sqrt{2}}{\sqrt{\pi}} \ln \sigma_{g}\right] \exp \left(4.5 \ln ^{2} \sigma_{g}\right),
\end{gathered}
$$

here $\gamma\left(a, \sigma_{g}\right)$ is airborne coefficient. Strictly speaking, because $\sigma_{g}$ is a function of $r_{g}$ and condensation nuclear, the character of $\gamma\left(a, \sigma_{g}\right)$ is not clear. Nothing but for simpleness, we specify $\gamma\left(a, \sigma_{g}\right)=\gamma$ (pending constant) in our study. Based on above discussion, with Equation (11), Equation (2) may be written as

$$
\begin{cases}-\frac{\mathrm{d}}{\mathrm{d} t}\left[\left(\frac{q}{q_{s}}\right)^{k} q_{s}\right]=\frac{\mathrm{d} m}{\mathrm{~d} t} & m<\gamma w^{1.5} q_{s} \\ -\frac{\mathrm{d} q_{s}}{\mathrm{~d} t}=\frac{\mathrm{d} m}{\mathrm{~d} t}+\eta & m=\gamma w^{1.5} q_{s}\end{cases}
$$

When liquid water precipitates, with Equation (13), a new formula of precipitate rate is got as

$$
\eta=-\frac{\mathrm{d} q_{\mathrm{s}}}{\mathrm{d} t}-\frac{\mathrm{d} \gamma w^{1.5} q_{\mathrm{s}}}{\mathrm{d} t}=-\frac{\mathrm{d}}{\mathrm{d} t}\left(\left(1+\gamma w^{1.5}\right) q_{\mathrm{s}}\right)
$$

From Equation (14), not only does precipitating liquid water contains condensed liquid water, but also contains converged and existing airborne liquid water.

In addition, Equation (14) can be rewritten as

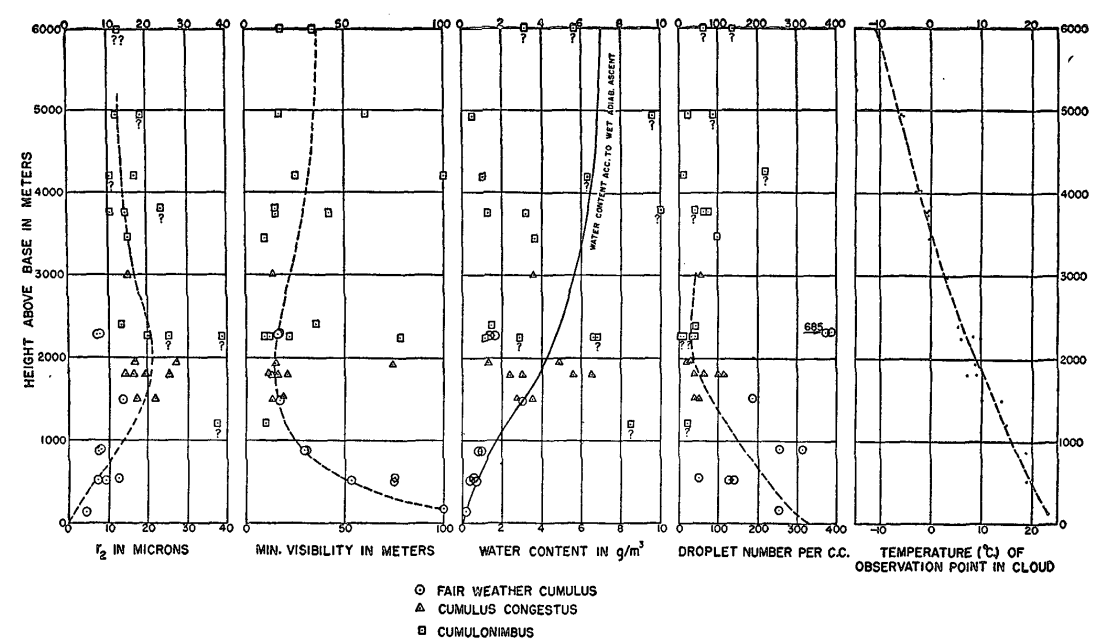

Figure 1. The physical properties in cumuliform clouds versus height above cloud base. 


$$
\eta=-\frac{\mathrm{d}}{\mathrm{d} t}\left(\left(1+\gamma w^{1.5}\right) q_{s}\right)=-\left(1+\gamma w^{1.5}\right) \frac{\mathrm{d} q_{s}}{\mathrm{~d} t}-1.5 q_{s} \gamma w^{0.5} \frac{\mathrm{d} w}{\mathrm{~d} t}
$$

The generalized temperature $T^{*}$ is defined as

$$
T^{*}=T \exp \left(\frac{L_{d}}{C_{p} T}\left(\frac{q}{q_{s}}\right)^{k} q_{s},\right.
$$

in saturated moist air ( $q=q_{s}$ ), Equation (15) can be rewritten as

$$
\eta=-\frac{C_{p} T}{L}\left(1+\gamma w^{1.5}\right) \frac{\mathrm{d} \ln \left(T^{\bullet} / T\right)}{\mathrm{d} t}-1.5 q_{s} \gamma w^{0.5} \frac{\mathrm{d} w}{\mathrm{~d} t} .
$$

In moist adiabatic condition, using thermodynamic equation, state equation and continuity,

$$
\begin{gathered}
C_{p} \frac{\mathrm{d} T}{\mathrm{~d} t}-\frac{1}{\rho} \frac{\mathrm{d} p}{\mathrm{~d} t}=-L_{d} \frac{\mathrm{d} q_{s}}{\mathrm{~d} t} \\
p=\rho R T \\
\frac{1}{\rho} \frac{\mathrm{d} \rho}{\mathrm{d} t}+\nabla V+\frac{\partial w}{\partial \mathrm{z}}=0,
\end{gathered}
$$

Equation (17) can be rewritten as

$$
\eta=\frac{C_{p} T}{L}\left(1+\gamma w^{1.5}\right) \nabla V+\frac{C_{p} T}{L}\left(1+\gamma w^{1.5}\right) \frac{\partial w}{\partial z}-1.5 q_{s} \gamma w^{0.5} \frac{\mathrm{d} w}{\mathrm{~d} t}
$$

In real atmosphere,

$$
\begin{gathered}
O\left(q_{s}\right) \leq 10^{1}(\mathrm{~g} / \mathrm{kg})=10^{-2} \\
O\left(\frac{\mathrm{d} w}{\mathrm{~d} t}\right) \leq O\left(w \frac{\partial w}{\partial z}\right) \\
\frac{C_{p} T}{L} \approx 2 \times 10^{-1}
\end{gathered}
$$

From Equation (22), (23) and (24), we have

$$
O\left(\frac{C_{p} T}{L}\left(\gamma w^{1.5}\right) \frac{\partial w}{\partial z}\right) \geq 10^{1} O\left(1.5 q_{s} \gamma w^{0.5} \frac{\mathrm{d} w}{\mathrm{~d} t}\right)
$$

In other words, $1.5 q_{s} \gamma w^{0.5} \frac{\mathrm{d} w}{\mathrm{~d} t}$ is negligible in Equation (15), (17) and (21), Equation (15), (17) and (21) can be rewritten as

$$
\begin{gathered}
\eta=-\left(1+\gamma w^{1.5}\right) \frac{\mathrm{d} q_{s}}{\mathrm{~d} t} \\
\eta=-\frac{C_{p} T}{L}\left(1+\gamma w^{1.5}\right) \frac{\mathrm{d} \ln \left(T^{\bullet} / T\right)}{\mathrm{d} t} \\
\eta=\frac{C_{p} T}{L}\left(1+\gamma w^{1.5}\right)\left(\nabla V+\frac{\partial w}{\partial z}\right)
\end{gathered}
$$

\subsection{The Discussion in the Condition of $w<0$}

In the condition of $w<0$, all liquid water always falls down immediately because of down-current, so

$$
m=m_{m}=0
$$




$$
\frac{\mathrm{d} m}{\mathrm{~d} t}=\frac{\mathrm{d} m_{m}}{\mathrm{~d} t}=0
$$

With Equation (30), Equation (2) becomes

$$
-\frac{\mathrm{d}}{\mathrm{d} t}\left[\left(\frac{q}{q_{s}}\right)^{k} q_{\mathrm{s}}\right]=\frac{\mathrm{d} m}{\mathrm{~d} t}+\eta=\eta
$$

From Equation (31), because $\eta \geq 0$, so

$$
\frac{\mathrm{d}}{\mathrm{d} t}\left[\left(\frac{q}{q_{s}}\right)^{k} q_{s}\right] \leq 0, \quad w<0
$$

In addition, because of down-current, the rising temperature cased by adiabatic expansion restrains condensation process, so

$$
\frac{\mathrm{d}}{\mathrm{d} t}\left[\left(\frac{q}{q_{\mathrm{s}}}\right)^{k} q_{\mathrm{s}}\right] \geq 0, \quad w<0
$$

From Equation (32) and (33), we have

$$
\frac{\mathrm{d}}{\mathrm{d} t}\left[\left(\frac{q}{q_{s}}\right)^{k} q_{\mathrm{s}}\right]=0, \quad w<0
$$

\section{The Improved Dynamic Equations on Non-Uniform Saturated Moist Atmosphere}

Following Wang X. R., Z. X. Wang and C. N. Shi (1999) [18] and above discussion, by introducing the condensation probability function $\left(q / q_{s}\right)^{k}$, specific water $m$ and maximum airborne specific water $m_{m}$, The improved dynamic equations on non-uniform saturated moist frictionless atmosphere in $\mathrm{z}$ coordinate may be provided as

$$
\begin{gathered}
\frac{\mathrm{d} V}{\mathrm{~d} t}+f K \times V=-\frac{1}{\rho} \nabla p \\
\frac{\mathrm{d} w}{\mathrm{~d} t}=-\frac{1}{\rho} \frac{\partial p}{\partial z}-g \\
C_{p} \frac{\mathrm{d} T}{\mathrm{~d} t}-\frac{1}{\rho} \frac{\mathrm{d} p}{\mathrm{~d} t}=-L_{d} \frac{\mathrm{d}}{\mathrm{d} t}\left[\left(\frac{q}{q_{s}}\right)^{k} q_{s}\right]+Q_{d} \\
p=\rho R T \\
\frac{1}{\rho} \frac{\mathrm{d} \rho}{\mathrm{d} t}+\nabla V+\frac{\partial w}{\partial z}=0 \\
-\frac{\mathrm{d}}{\mathrm{d} t}\left(\left(\frac{q}{q_{s}}\right)^{k} q_{s}\right)=0 \quad w<0 \\
-\frac{\mathrm{d}}{\mathrm{d} t}\left(\left(\frac{q}{q_{s}}\right)^{k} q_{s}\right)=\frac{\mathrm{d} m}{\mathrm{~d} t} \quad w \geq 0, m<m_{m}=\gamma w^{1.5} q_{s} \\
-\frac{\mathrm{d} q_{s}}{\mathrm{~d} t}=\frac{\mathrm{d} m}{\mathrm{~d} t}+\eta \quad w \geq 0, m=m_{m}=\gamma w^{1.5} q_{s}
\end{gathered}
$$




$$
\left\{\begin{array}{lc}
\eta=0 & w<0 \\
\eta=0 & w \geq 0, m<m_{m}=\gamma w^{1.5} q_{s} \\
\eta=-\frac{\mathrm{d}}{\mathrm{d} t}\left(1+\gamma w^{1.5}\right) q_{s} \cong-\left(1+\gamma w^{1.5}\right) \frac{\mathrm{d} q_{s}}{\mathrm{~d} t} & w \geq 0, m=m_{m}=\gamma w^{1.5} q_{s}
\end{array}\right.
$$

In moist adiabatic condition, from Equations (35)-(41) and the definition of generalized temperature $T^{*}$ (16), built upon the transform formula between $\omega$ (in $p$ coordinate) and $w$ (in z coordinate)

$$
-\omega=\frac{p}{R T} g w
$$

and the vertical coordinate transform formula based on the non-static equilibrium by Wang X. R., C. E. Chi and Z. X. Wang (1997) [17], the improved dynamic equations on non-uniform saturated moist frictionless and adiabatic atmosphere in $p$ coordinate may be provided as

$$
\begin{aligned}
& \frac{\mathrm{d} V}{\mathrm{~d} t}+f K \times V=-\frac{T}{T^{*}} \nabla \Phi \\
& \frac{\partial \Phi}{\partial p}=-\frac{R T^{*}}{p} \\
& C_{p} \frac{\mathrm{d} T^{*}}{\mathrm{~d} t}+p \frac{\mathrm{d}\left(\frac{R T^{*}}{p}\right)}{\mathrm{d} t}=0 \\
& \frac{\mathrm{d} \ln \left(T^{*} / T\right)}{\mathrm{d} t}+\nabla V+\frac{\partial \omega}{\partial p}=0 \\
& \frac{\mathrm{d}^{2} \Phi}{\mathrm{d} t^{2}}=\left(\frac{T}{T^{*}}-1\right) g^{2} \\
& \int-\frac{\mathrm{d}}{\mathrm{d} t}\left(\left(\frac{q}{q_{s}}\right)^{k} q_{s}\right)=0 \quad \omega>0 \\
& \left\{-\frac{\mathrm{d}}{\mathrm{d} t}\left(\left(\frac{q}{q_{s}}\right)^{k} q_{s}\right)=\frac{\mathrm{d} m}{\mathrm{~d} t} \quad \omega \leq 0, m<m_{m}=\gamma\left(-\omega \frac{R T}{p g}\right)^{1.5} q_{s}\right. \\
& -\frac{\mathrm{d} q_{s}}{\mathrm{~d} t}=\frac{\mathrm{d} m}{\mathrm{~d} t}+\eta \quad \omega \leq 0, m=m_{m}=\gamma\left(-\omega \frac{R T}{p g}\right)^{1.5} q_{s} \\
& \left\{\begin{array}{lc}
\eta=0 & \omega>0 \\
\eta=0 & \omega \leq 0, m<m_{m}=\gamma\left(-\omega \frac{R T}{p g}\right)^{1.5} q_{s} \\
\eta=-\frac{\mathrm{d}}{\mathrm{d} t}\left(1+\gamma\left(-\omega \frac{R T}{p g}\right)^{1.5}\right) q_{s}=-\left(1+\gamma\left(-\omega \frac{R T}{p g}\right)^{1.5}\right) \frac{\mathrm{d} q_{s}}{\mathrm{~d} t} & \omega \leq 0, m=m_{m}=\gamma\left(-\omega \frac{R T}{p g}\right)^{1.5} q_{s}
\end{array}\right.
\end{aligned}
$$

From Equation (35)-(41) and (43)-(49), the dynamic equations on non-uniform saturated moist atmosphere are improved, because of introducing specific water $m$ and maximum airborne specific water $m_{m}$, and eliminating the irrational supposition that condensed liquid water always falls immediately, and adding the balance 
equation between water vapor and airborne liquid water (40) or (48) and the new formula of precipitate rate (41) or (49). From Equation (40) or (48), in up-current $(w \geq 0)$ or $(\omega \leq 0)$, the condensed liquid water can precipitate under the condition with $q=q_{s}$ and $m=m_{m}=\gamma w^{1.5} q_{s}$ or $m=m_{m}=\gamma(-\omega R T / p g)^{1.5} q_{s}$ only, otherwise it is detained in the air and becomes airborne liquid water. From Equation (41) or (49), when liquid water precipitates, not only does precipitating liquid water contain condensed liquid water, but also contains converged and existing airborne liquid water.

\section{The Rough Estimation of Airborne Coefficient}

Because the character of $\gamma\left(a, \sigma_{g}\right)$ is not clear, its accurate calculation requires a large number of data and complex work, so it is to be discussed in another article on account of space relation. Here, it is supposed that $\gamma\left(a, \sigma_{g}\right)$ is a constant (airborne coefficient $\gamma$ ) only.

With the new formula of precipitate rate in $p$ coordinate (49), the new formula of rainfall intensity can be written roughly as

$$
I_{a}=-\frac{1}{g} \int_{c b}^{c t} \frac{\mathrm{d}}{\mathrm{d} t}\left(1+\gamma\left(-\omega \frac{R T}{p g}\right)^{1.5}\right) q_{s} \mathrm{~d} p
$$

where $I_{a}$ is the new estimated value of rainfall intensity (containing condensed liquid water and converged existing airborne liquid water), $c_{b}$ is the height of cloud base, $c_{t}$ is the height of cloud top.

When estimating rainfall intensity, it is usually assumed that the local change of meteorological equal to 0 , so

$$
\frac{\mathrm{d}}{\mathrm{d} t}\left(1+\gamma\left(-\omega \frac{R T}{p g}\right)^{1.5}\right) q_{\mathrm{s}}=\vec{V} \cdot\left[\left(1+\gamma\left(-\omega \frac{R T}{p g}\right)^{1.5}\right) q_{\mathrm{s}}\right]+\omega \frac{\partial}{\partial p}\left[\left(1+\gamma\left(-\omega \frac{R T}{p g}\right)^{1.5}\right) q_{s}\right]
$$

If assuming $\vec{V} \cdot\left[\left(1+\gamma\left(-\omega \frac{R T}{p g}\right)^{1.5}\right) q_{s}\right]$ as a quadratic function and considering $\omega_{c t}=0$, then, according Zhang et al., (1990) [41], from (50) and (51), there is:

$$
I_{a}=I_{c}+\gamma F C
$$

where $I_{c}$ is the estimated value by the conventional rainfall intensity formula, it may be expressed as

$$
I_{c}=\frac{1}{g}\left\{\frac{c t-c b}{6}\left[\nabla \cdot\left[\overrightarrow{V_{c b}} q_{s c b}\right]+4 \nabla \cdot\left[\overrightarrow{V_{c m}} q_{s c m}\right]+\nabla \cdot\left[\overrightarrow{V_{c t}} q_{s c t}\right]\right]-\frac{\omega_{c b} R T_{c b}}{c b \cdot g} q_{s c b}\right\}
$$

here

$$
c m=0.5(c t+c b) \text {. }
$$

where $F c$ is the confluent function of airborne liquid water, it may be expressed as

$$
\begin{gathered}
F c=\frac{1}{g}(A+B+C) \\
A=\left(-\frac{\omega_{c b} R T_{c b}}{c b \cdot g}\right)^{2.5} q_{s c b} \\
B=\frac{c t-c b}{6} \nabla \cdot\left[\overrightarrow{V_{c b}}\left(-\frac{\omega_{c b} R T_{c b}}{c b \cdot g}\right)^{1.5} q_{s c b}\right] \\
C=2 \frac{c t-c b}{3} \nabla \cdot\left[\overrightarrow{V_{c m}}\left(-\frac{\omega_{c m} R T_{c m}}{c m \cdot g}\right)^{1.5} q_{s c m}\right]
\end{gathered}
$$

On the theoretical plane, using the synchronous analysis data with rainfall provided by RAFS (regional analy- 
sis and forecasting system), let the actual rainfall intensity $I_{0}=I_{a}$, the $\gamma$ could be calculated from (52-58), but, in fact, it is very difficult, because, the error of $\omega_{c b}$ and $\omega_{c m}$ (caused by the restrictions of observation systems) is so big that, the error of estimated $I_{c}$ is also big, and it amounts to or exceed $\gamma F c$ frequently, and sometimes $I_{c}>I_{0}$. Only when the actual rainfall intensity is strong enough before $\gamma$ can be estimated.

Here, in the supposition that $\gamma\left(a, \sigma_{g}\right)$ is a constant (airborne coefficient $\gamma$ ) only, using the data provided by the leading group for studying the super-heavy rain in Henan in China in August 4-8 1975 [LGSHR1975], (1977) [42], $\gamma$ is estimated roughly.

The gross-precipitation of this super-heavy rain is $1631.1 \mathrm{~mm}$, the maximum rainfall intensity is $189.5 \mathrm{~mm} / \mathrm{h}$ (in the neighborhood of Banqiao reservoir in August 7, 22:00 Beijing time), in August 7, 20:00 Beijing time (the observation time on schedule), the center rainfall intensity on large scale surface weather chart is $12.5 \mathrm{~mm} / \mathrm{h}$, the actual extremes of rainfall intensity is $99.7 \mathrm{~mm} / \mathrm{h}$ (in the neighborhood of Banqiao reservoir). Although the horizontal distribution graph of $I_{0}$ (the rainfall intensity estimated by the traditional formula of precipitate rate) is very similar to actual distribution graph (figures not shown), the center value of $I_{0}$ is $7.7 \mathrm{~mm} / \mathrm{h}$, which is much less than actual center value $12.5 \mathrm{~mm} / \mathrm{h}$ not mentioning the actual extremes of rainfall intensity $99.7 \mathrm{~mm} / \mathrm{h}$.

With the new formula of precipitate rate in $p$ coordinate (49), using mean value theorem, the new formula of rainfall intensity can be written roughly as

$$
I_{n}=-\frac{1}{g} \int_{c b}^{c t}\left(1+\gamma\left(-\omega \frac{R T}{p g}\right)^{1.5}\right) \frac{\mathrm{d} q_{s}}{\mathrm{~d} t} \mathrm{~d} p \approx-\frac{1}{g} \overline{\left(1+\gamma\left(-\omega \frac{R T}{p g}\right)^{1.5}\right)} \int_{c b}^{c t} \frac{\mathrm{d} q_{s}}{\mathrm{~d} t} \mathrm{~d} p \approx\left(1+\gamma \overline{\left(-\omega \frac{R T}{p g}\right)^{1.5}}\right) I_{o}
$$

where $I_{n}$ is the new estimated value of rainfall intensity (containing condensed liquid water and converged existing airborne liquid water), $c_{b}$ is the height of cloud base, $c_{t}$ is the height of cloud top, $\overline{-\omega R T / p g}$ is the mean value of $-\omega R T / p g$. Since rainfall is from condensed liquid water and converged and existing airborne liquid water in lower level, $\overline{-\omega R T / p g}$ may be replaced by the mean value at $900 \mathrm{hPa}$ and $500 \mathrm{hPa}$, we have

$$
-\omega \frac{R T}{p g}=\frac{1}{2}\left(-\frac{\omega_{900}}{900}-\frac{\omega_{500}}{500}\right) \frac{R \bar{T}}{g}
$$

From the analysis report provided by LGSHR1975 (1977) [42], let the center value of $I_{n}$ equal to actual center value $12.5 \mathrm{~mm} / \mathrm{h}$, the center value of $I_{0}$ is $7.7 \mathrm{~mm} / \mathrm{h}, R=2.87 \mathrm{hPa} \cdot \mathrm{m}^{3} \cdot \mathrm{kg}^{-1} \cdot \mathrm{K}^{-1}, g=9.8 \mathrm{~m} \cdot \mathrm{s}^{-2}, \quad-\omega_{900}=$ $7 \times 10^{-3} \mathrm{hPa} / \mathrm{s}, \quad-\omega_{500}=5 \times 10^{-3} \mathrm{hPa} / \mathrm{s}$ and $\bar{T}=293 \mathrm{~K}$ at the center of rainfall intensity, with equation (59) and (60), we get

$$
\gamma \approx 0.95 m^{=1.5} \mathrm{~s}^{1.5}
$$

Based on $8 \mathrm{~m} / \mathrm{s}$ (the surface wind velocity (SWV) recorded at Suiping weather station, which is the nearest from Banqiao reservoir) and $12 \mathrm{~m} / \mathrm{s}$ (the SWV estimated from the physical phenomena at Banqiao reservoir), with an eye on the small scale orographic influence of Banqiao reservoir (climbing and bell-mouthed effects in the condition of NE wind), the $I_{0},-\omega_{900}$ and $-\omega_{500}$ at Banqiao reservoir are given by LGSHR1975 (1977) [42] (see the Table 1). From these data and $\gamma \approx 0.95 m^{=1.5} s^{1.5}$, with Equation (49) and (50), we can get the $I_{n}$ at Banqiao reservoir (see the Table 1).

From Table 1, although the traditional estimated values of rainfall intensity at Banqiao reservoir, $I_{0}=30.7$ $\mathrm{mm} / \mathrm{h}$ and $I_{0}=37.0 \mathrm{~mm} / \mathrm{h}$ (based on SMV $=8 \mathrm{~m} / \mathrm{s}$ and $12 \mathrm{~m} / \mathrm{s}$ respectively), are much less than the actual extremes of rainfall intensity $99.7 \mathrm{~mm} / \mathrm{h}$, the new estimated values $I_{n}=65.1 \mathrm{~mm} / \mathrm{h}$ and $81.8 \mathrm{~mm} / \mathrm{h}$ (based on $\mathrm{SMV}=8 \mathrm{~m} / \mathrm{s}$ and $12 \mathrm{~m} / \mathrm{s}$ respectively), are very close to the actual extremes of rainfall intensity $99.7 \mathrm{~mm} / \mathrm{h}$.

\section{Conclusions}

In this study, by introducing specific water and maximum airborne specific water into the dynamic framework on non-uniform saturated moist atmosphere, the irrational supposition, that condensed liquid water always falls immediately, is eliminated. The balance equation between water vapor and airborne liquid water is derived. From the balance equation, a new formula of precipitate rate is derived. Following the above discussion, improved dynamic equations on non-uniform saturated moist atmosphere are provided. Finally, in the supposition that $\gamma\left(a, \sigma_{g}\right)$ is a constant (airborne coefficient $\gamma$ ) only, using the data provided by the leading group for studying the super-heavy rain in Henan in China in August 4-8, 1975 [LGSHR1975], (1977) [42], $\gamma$ is estimated roughly. 
Table 1. The $I_{0},-\omega_{900}$ and $-\omega_{500}$ are given by LGSHR1975 (1977) [42] and the $I_{n}$ estimated from the new formula of rainfall intensity at Banqiao reservoir.

\begin{tabular}{ccccc}
$\mathrm{SWV}$ & $\begin{array}{c}-\omega_{900} \\
10^{-3} \mathrm{hPa} / \mathrm{s}\end{array}$ & $\begin{array}{c}-\omega_{500} \\
10^{-3} \mathrm{hPa} / \mathrm{s}\end{array}$ & $\begin{array}{c}I_{o} \\
\mathrm{~mm} / \mathrm{h}\end{array}$ & $\begin{array}{c}I_{n} \\
\mathrm{~mm} / \mathrm{h}\end{array}$ \\
$\mathrm{m} / \mathrm{s}$ & 15 & 5 & 30.7 & 65.1 \\
12 & 16.4 & 5 & 37.0 & 81.8 \\
\hline
\end{tabular}

It is necessary to point out that the character of $\gamma\left(a, \sigma_{g}\right)$ is not clear, and its accurate calculation requires a large number of data and complex work. By and large, there is still much work to estimate $\gamma$ accurately.

\section{Acknowledgements}

This study was supported by Anhui Provincial Natural Science Foundation under Grant No. 1508085MD64, 1408085MKL60 and China Meteorology Administration Foundation under Grant No. CMAGJ2015M28.

\section{References}

[1] Betts, A.K. (1973) Non Precipitating Cumulus Convection and Its Parameterization. Quarterly Journal of the Royal Meteorological Society, 99, 178-196. http://dx.doi.org/10.1002/qj.49709941915

[2] Xie, Y.B. (1978) Synoptic Problems in Moist Baroclinic Atmosphere. In: Thesis Collection on Torrential Rain, Jilin Press, Changchun, 1-15. (In Chinese)

[3] Wang, L.M. (1980) The Basic Equations and Main Characteristics of Saturated Moist Dynamics. Acta Mechanica Sinica, 38, 44-50. (In Chinese)

[4] Wang, L.M. (1980) The Basic Characteristics of Saturated Moist Thermodynamic. Acta Mechanica Sinica, 38, 106109. (In Chinese)

[5] Tripoli, G.J. and Cotton, W.R. (1981) The Use of Liquid Water Potential Temperature as a Thermodynamic Variable in Deep Atmospheric Models. Monthly Weather Review, 109, 1094-1102. http://dx.doi.org/10.1175/1520-0493(1981)109<1094:TUOLLW>2.0.CO;2

[6] Pointin, Y. (1984) Wet Equivalent Potential Temperature and Enthalpy as Prognostic Variables in Cloud Modeling. Journal of the Atmospheric Sciences, 41, 651-660. http://dx.doi.org/10.1175/1520-0469(1984)041<0651:WEPTAE>2.0.CO;2

[7] Hauf, T. and Holler, H. (1987) Entropy and Potential Temperature. Journal of the Atmospheric Sciences, 44, 28872901. http://dx.doi.org/10.1175/1520-0469(1987)044<2887:EAPT>2.0.CO;2

[8] Ooyama, K.V. (1990) A Thermodynamic Foundation for Modeling the Moist Atmosphere. Journal of the Atmospheric Sciences, 47, 2580-2593. http://dx.doi.org/10.1175/1520-0469(1990)047<2580:ATFFMT>2.0.CO;2

[9] Ooyama, K.V. (2000) A Dynamic and Thermodynamic Foundation for Modeling the Moist Atmosphere with Parameterized Microphysics. Journal of the Atmospheric Sciences, 58, 2073-2102. http://dx.doi.org/10.1175/1520-0469(2001)058<2073:ADATFF>2.0.CO;2

[10] Persson, O. (1995) Simulations of the Potential Vorticity Structure and Budget of FRONTS 87 IOP8. Quarterly Journal of the Royal Meteorological Society, 121, 1041-1081. http://dx.doi.org/10.1002/qj.49712152506

[11] Wu, G.X., Cai, Y.P. and Tang, X.J. (1995) Moist Potential Vorticity and Slantwise Vorticity Development. Acta Meteor Simica, 53, 387-405. (In Chinese)

[12] Soriano, L.J. and Garcia Diez, E.L. (1997) Effect of Ice on the Generation of a Generalized Potential Vorticity. Journal of the Atmospheric Sciences, 54, 1385-1387. http://dx.doi.org/10.1175/1520-0469(1997)054<1385:EOIOTG>2.0.CO;2

[13] Schubert, W.H., Hausman, S.A., Garcia, M., Ooyama, K.V. and Kuo, H.-C. (2001) Potential Vorticity in a Moist Atmosphere. Journal of the Atmospheric Sciences, 58, 3148-3157. http://dx.doi.org/10.1175/1520-0469(2001)058<3148:PVIAMA>2.0.CO;2

[14] Bannon, P.R. (2002) Theoretical Foundations for Models of Moist Convection. Journal of the Atmospheric Sciences, 59, 1967-1982. http://dx.doi.org/10.1175/1520-0469(2002)059<1967:TFFMOM>2.0.CO;2

[15] Mason, N.B. (1971) The Physics of Clouds. Oxford University Press, Oxford, 671.

[16] Wang, X.R. and Wu, K.J. (1995) An Approach to the Dynamics of Moist Air. Scientia Meteorologica Sinica, 15, 9-17. (In Chinese)

[17] Wang, X.R., Chi, C.E. and Wang, Z.X. (1997) The Vertical Coordinate Transformation and the Dynamics Equations of Moist Air Based on the Non-Static Equilibrium. Scientia Meteorologica Sinica, 21, 557-563. (In Chinese) 
[18] Wang, X.R., Wang, Z.X. and Shi, C.N. (1999) The Introduction of Condensation Probability Function and the Dynamic Equations on Non-Uniform Saturated Moist Air. Journal of Tropical Meteorology, 15, 64-70. (In Chinese)

[19] Wang, X.R., Wang, Z.X. and Shi, C.N. (1998) On the Non-Conservativeness of Wet Potential Vorticity of Air Motion. Scientia Meteorologica Sinica, 13, 136-141. (In Chinese)

[20] Gao, S.T., Wang, X.R. and Zhou, Y.S. (2004) Generation of Generalized Moist Potential Vorticity in a Frictionless and Moist Adiabatic Flow. Geophysical Research Letters, 31, Article ID: L12113. http://dx.doi.org/10.1029/2003gl019152

[21] Wang, X.R. and Wei, M. (2007) Theoretical Analysis of Non-Uniform Saturated Moist Potential Vorticity (NUSMPV) Associated with the Occurrence and Development of Weather Systems. Journal of Tropical Meteorology, 23, 459-566. (In Chinese)

[22] Wang, X.R., Chen, X.P. and Xun, S.P. (1998) Study on Sudden Disaster Based on Trigger Mechanism of Unstable Energy. Journal of Natural Disasters, 7, 11-15. (In Chinese)

[23] Wang, X.R., Wu, K.J., Chen, X.P. and Shi, C.E. (1999) Dynamic Mechanism of the Happening of the Sudden Calamitous Weather. Journal of Nanjing Institute of Meteorology, 22, 711-715. (In Chinese)

[24] Wang, X.R., Zheng, Y.Y., Gao, S.T. and Zhou, K. (2006) Possible Premonitory Characters of Doppler Radar Product and Cloud Chart for the Mid-Latitude Sudden Heavy Rain. Journal of Tropical Meteorology, 22, 612-617. (In Chinese)

[25] Gao, S.T., Zhou, Y.S., Lei, T., et al. (2005) Analyses of Hot and Humid Weather in Beijing City in Summer and Its Dynamical Identification. Science in China, Ser. D, Earth Sciences, 48, 128-137.

[26] Sheng, S.Q. and Zhou, Y.S. (2007) Advances in Meso-Scale Dynamical Analysis of Torrential Rain Systems in Recent Years in China. Chinese Journal of Atmospheric Sciences, 31, 1171-1188. (In Chinese)

[27] Yang, S., Gao, S.T. and Wang, D.H. (2007) Diagnostic Analysis of the Ageostrophic Q Vector in the Non-Uniformly Saturated, Frictionless and Moist Adiabatic Flow. Journal of Geophysical Research, 112, Article ID: D09114. http://dx.doi.org/10.1029/2006JD008142

[28] Zhou, Y.S., Cao, S.J. and Wang, D.H. (2007) Application of Generalized Moist Potential Vorticity in Non-Uniformly Saturated Atmosphere to Torrential Rain Forecast. Journal of Applied Meteorological Science, 18, 754-759. (In Chinese)

[29] Duan, T.Y., Deng, G. and Wang, D.H. (2007) Diagnostic Analysis of Generalized Moist Potential Vorticity and Forecast of Torrential Rain Region. Chinese Journal of Atmospheric Sciences, 31, 1301-1307. (In Chinese)

[30] Gao, S.T. and Cui, C.G. (2007) The Theory of Generalized Moist Potential Vorticity and Its Applicative Study. Torrential Rain and Disasters, 26, 5-10. (In Chinese)

[31] Cao, J. and Gao, S.T. (2008) Generalized Potential Temperature in Non-Uniformly Saturated Atmosphere. Chinese Journal of Geophysics, 51, 1651-1656. (In Chinese)

[32] Zhou, Y.S. (2009) Application of Generalized Moist Potential Vorticity to Analysis and Forecast of Torrential Rain over the Chang Jiang-Huai He River Basin. Chinese Journal of Atmospheric Sciences, 33, 1101-1110. (In Chinese)

[33] Liang, Z.M., Lu, C.G. and Tollerud, E.I. (2010) Diagnostic Study of Generalized Moist Potential Vorticity in a Non-Uniformly Saturated Atmosphere with Heavy Precipitation. Quarterly Journal of the Royal Meteorological Society, 136, 1275-1288. http://dx.doi.org/10.1002/qj.636

[34] Zhou, Y.S. and Zhu, K.F. (2010) Comparisons between the Generalized Potential Temperature in the Moist Atmosphere and the Potential Temperature in the Dry Atmosphere and the Equivalent Potential Temperature in the Saturated Moist Atmosphere. Acta Meteor Simica, 68, 612-616. (In Chinese)

[35] Wang, X.R. and Gao, S.T. (2007) The Reestimate of Rain Intensity Extremes of the Super-Heavy Rain in Henan in China in Aug.1975. Journal of Tropical Meteorology, 23, 196-200. (In Chinese)

[36] Levin, L.M. (1954) Distribution Function of Cloud and Raindrops by Sizes. Doklady Akademii Nauk SSSR, $94,1045$.

[37] Squires, P. (1958) The Spatial Variation of Liquid Water Content and Droplet Concentration in Cumuli. Tellus, 10, 372-380. http://dx.doi.org/10.1111/j.2153-3490.1958.tb02024.x

[38] Warner, J. (1955) The Water Content of Cumuliform Cloud. Tellus, 7, 449-457. http://dx.doi.org/10.1111/j.2153-3490.1955.tb01183.x

[39] Warner, J. and Newnham, T.D. (1952) A New Method of Measurement of Cloud Water Content. Quarterly Journal of the Royal Meteorological Society, 78, 46-52. http://dx.doi.org/10.1002/qj.49707833506

[40] Weickmann, H.K. and Aufm Kampe, H.J. (1953) Physical Properties of Cumulus Clouds. Journal of the Meteorology, 10, 204-211. http://dx.doi.org/10.1175/1520-0469(1953)010<0204:ppocc >2.0.c0;2

[41] Zhang, Y., et al. (1990) The Forecast of Heavy Rain. Meteorological Press, Beijing, 424. (In Chinese)

[42] The Leading Group for Studying the Super-Heavy Rain in Henan in China in August 4-8 1975 (LGSHR1975) (1977) The Analysis Report on the Super-Heavy Rain in Henan in China in August 4-8 1975. (In Chinese) 